\title{
Study on Transformation of Ginsenosides in Different Methods
}

\author{
Meng-meng Zheng, Fang-xue Xu, Yu-juan Li, Xiao-zhi Xi, Xiao-wei Cui, \\ Chun-chao Han, and Xue-lan Zhang
}

School of Pharmacy, Shandong University of Traditional Chinese Medicine, Jinan 250355, China

Correspondence should be addressed to Chun-chao Han; chunchaoh@126.com and Xue-lan Zhang; zhang8832440@sina.com

Received 19 September 2017; Revised 6 November 2017; Accepted 20 November 2017; Published 14 December 2017

Academic Editor: Gail B. Mahady

Copyright ( 2017 Meng-meng Zheng et al. This is an open access article distributed under the Creative Commons Attribution License, which permits unrestricted use, distribution, and reproduction in any medium, provided the original work is properly cited.

\begin{abstract}
Ginseng is a traditional Chinese medicine and has the extensive pharmacological activity. Ginsenosides are the major constituent in ginseng and have the unique biological activity and medicinal value. Ginsenosides have the good effects on antitumor, antiinflammatory, antioxidative and inhibition of the cell apoptosis. Studies have showed that the major ginsenosides could be converted into rare ginsenosides, which played a significant role in exerting pharmacological activity. However, the contents of some rare ginsenosides are very little. So it is very important to find the effective way to translate the main ginsenosides to rare ginsenosides. In order to provide the theoretical foundation for the transformation of ginsenoside in vitro, in this paper, many methods of the transformation of ginsenoside were summarized, mainly including physical methods, chemical methods, and biotransformation methods.
\end{abstract}

\section{Introduction}

Ginseng (Panax ginseng Meyer) is a traditional Chinese medicine, which is used in ancient eastern countries. Ginseng is used as energy booster and can fortify the spleen to benefit the lungs, nourish fluids, calm the heart, tranquilize the mind, and so on [1-3]. Ginsenosides and polysaccharides are the main active substances in ginseng. Studies on the ginseng polysaccharides are few [4-6]. But there are a lot of reports about ginsenosides. Ginseng has so many pharmacological activities because of ginsenosides. According to the difference in the position and quantity of sugar moiety in the glycosides, ginsenosides are divided into three types: protopanaxadiol (PPD), protopanaxatriol (PPT), and oleanolic acid [7, 8]. At present, 60 ginsenosides approximately were isolated and identified from family Araliaceae. Ginsenosides showed many bioactive and pharmacological activities, such as anticancer, anti-inflammatory, antiaging, and antioxidant [9-12]. In addition, some researchers suggested that minor ginsenoside and aglycone are superior to the major ginsenoside at antitumor [13]. The antitumor activity is decreasing with the increasing sugar moiety in the glycosides [14]. But the contents of minor ginsenoside and aglycone are low or few in ginseng. Minor ginsenosides extracted from plants cannot satisfy the scientific researches and the clinical requirements. So it is very significant to prepare the minor ginsenoside through transforming the major ginsenoside. In recent years, the researches about the conversion of ginsenosides have obtained many significant results and studies on the transformation mechanism also made some progress $[15,16]$.

In this paper, we summarized many methods of the transformation of ginsenosides, mainly including physical methods, chemical methods, and biotransformation (Figure 1). The purpose of this study is to provide the theoretical basis for the new methods and approaches for the transformation and biosynthesis of ginsenosides.

\section{Physical Methods}

2.1. Steaming Transformation. Heat-treatment of ginseng is a conventional way that is widely applied in ancient times. Ginseng could product some rare ginsenosides when heated at a higher temperature than conventional preparation. To study on the transformation induced by steaming, red ginseng samples were prepared by steaming of white ginseng at $98^{\circ} \mathrm{C}$ for $3 \mathrm{~h}$ or $1 \mathrm{~h}$ and drying at $60^{\circ} \mathrm{C}$ for $12 \mathrm{~h}$. The results proved 


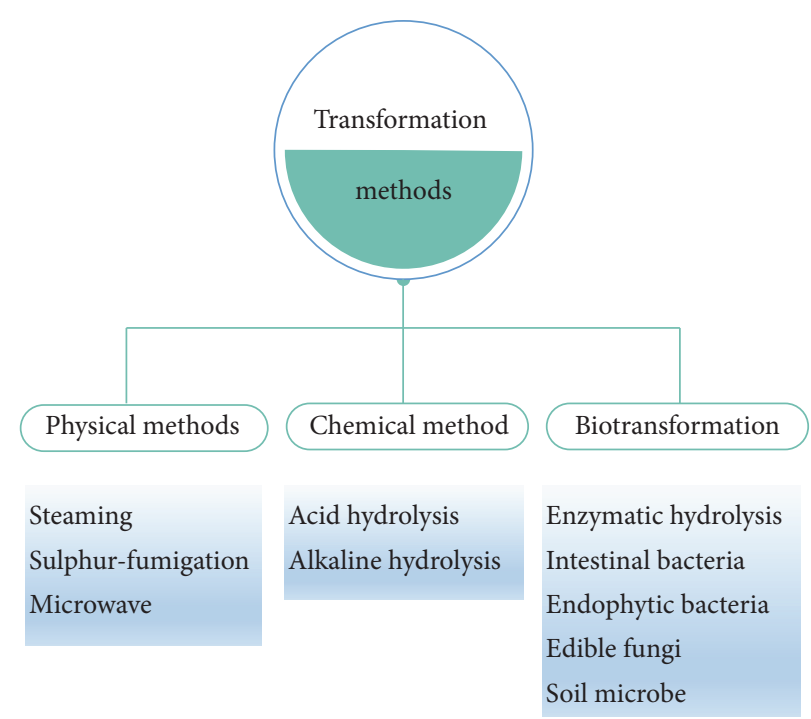

FiguRE 1: Methods applied on the transformation of ginsenosides. Three types of methods were concluded, which included the physical methods, chemical method, and biotransformation. Each type of method contained several kinds of common method that transformed a ginsenoside into another kind of ginsenoside by changing ginsenoside's chemical structure.

that some malonyl ginsenosides were generated in the heated white ginseng, while they were not detected in the steaming ginseng, and the contents of malonyl ginsenosides would be decreased during steaming. This change might be related to enzymes which convert malonyl ginsenosides to corresponding neutral ginsenosides [17]. Chemical constituents and biological activities of ginseng would be changed when ginseng was steamed at a temperature over $100^{\circ} \mathrm{C}$. After steaming at $120^{\circ} \mathrm{C}$, some ginsenosides were produced, such as ginsenoside $\mathrm{Rg} 3, \mathrm{~F} 4$, and $\mathrm{Rg} 5$, which did not exist at raw ginseng. But the contents of ginsenoside $\mathrm{Rf}, \mathrm{Re}, \mathrm{Rd}$, $\mathrm{Rc}, \mathrm{Rb} 1$, and $\mathrm{Rb} 2$ were decreased after steaming and were fewer when steamed at $120^{\circ} \mathrm{C}$ [18]. Besides, when ginseng steamed at $120^{\circ} \mathrm{C}$ for $3 \mathrm{~h}$, the contents of some ginsenosides in the methanol extract were showed as follows: 20(S)Rg3 (0.700\%), 20(R)-Rg3 (0.643\%), Rg5 (0.492\%), and other saponins (1.37\%) [19]. In addition, an autoclave was used to steam the root of fresh ginseng, and HPLC was used to defected the change of ginseng's constituents. On the one hand, temperature has a significant influence on the conversion of ginsenosides. For example, the contents of total ginsenosides were increased after ginseng roots were steamed at $98^{\circ} \mathrm{C}$ and decreased gradually at $120^{\circ} \mathrm{C}$. On the other hand, time was also important. For instance, the contents of Rb1, Rb2, Rc, and Rd were increased sharply within first $0.5 \mathrm{~h}$ at $120^{\circ} \mathrm{C}$ and almost disappeared after $4 \mathrm{~h}$. Moreover, acidic ginsenosides and neutral ginsenosides would be converted to rare ginsenosides during the root steaming. Acidic ginsenosides play an important role in the transformation of ginsenosides because they can release malonic acid and acetic acid, which affect the process of the transformation of saponins [20]. What is more, the combination of resin adsorption and heating may have a better application in conversion of ginsenosides. Total ginsenosides were obtained by $70 \%(\mathrm{v} / \mathrm{v})$ ethanol and loaded to HP-20 open column.
The ginsenosides enriched fractions were eluted by $65 \%$ methanol. The extraction from HP-20 was heated in autoclave at $130^{\circ} \mathrm{C}$ for $4 \mathrm{~h}$, and the steam-treated extraction was separated by AB-8 and identified using HPLC-APLC-MS. The result showed that polar ginsenosides can convert to less polar ones, and PPD group could translate into $20(\mathrm{~S}) /(\mathrm{R})$ Rg3 by selectively eliminating the C-20 sugar chain at high temperature and pressure. The transformed pathways of some ginsenosides by heat were as follows: Rb1, Rb2, Rc, Rd $\rightarrow$ Rg3 $\rightarrow$ Rk1, Rg5, Rs3; Re $\rightarrow \operatorname{Rg} 2 \rightarrow \operatorname{Rg} 6$ and F4; Rg1 $\rightarrow$ Rh1 [21].

2.2. Sulphur-Fumigation Transformation. Not only sunlight or heating is traditional method to process ginseng, but also sulphur-fumigation is also a convenient way to handle ginseng and other medicinal herbs. The slices of ginseng and sulphur powder were put into a desiccator at the lower and upper layer at $25^{\circ} \mathrm{C}$ for $12 \mathrm{~h}$, respectively. The sulphurfumigated ginseng was extracted by $75 \%$ ethanol at $85^{\circ} \mathrm{C}$ for $3 \mathrm{~h}$. The experimental results suggested that some new compounds appeared in sulphur-fumigated ginseng comparing with reference compounds and nonfumigated ginseng by using UPLC-QTOF-MS/MS, such as ginsenosides Rh2 and Rg5 [22]. Some ginsenosides can be converted to ginsenoside derivatives or sulfate in sulphur-fumigation process through hydrolysis, dehydration, and decarboxylation [22-24]. Furthermore, sulphur-fumigation can influence the contents of original ginsenosides. For example, the contents of Rg1, Re, $\mathrm{Rc}, \mathrm{Rb} 2$, and Rd were all decreased after sulphur-fumigation. The total contents of 10 ginsenosides in sulphur-fumigated white ginseng were decreased by up to $64 \%$ comparing with nonfumigated white ginseng [22]. Sulphur-fumigated white ginseng from different country could be discriminated by the chemical fingerprint of sulphur-fumigated ginseng. This also provides the useful way to identify other sulphur-fumigated medicinal herbs [23]. 
2.3. Microwave Transformation. The traditional processing methods of ginseng include conventional heating and sulphur-fumigation, but they have some drawbacks. The heating process is slow and the sulphur-fumigation would produce some hazardous substance. Recently, microwave heating has been applied on natural products and microwave heating is a simple, efficient, and time saving way to process products. The floatation solution of ginseng was put into the microwave irradiation system and the degradation of ginsenosides was continuously performed for $5 \mathrm{~min}$ under $165^{\circ} \mathrm{C}$. The solutions after degradation were extracted by water-saturated n-butanol and evaporated to dryness. The results showed that ginsenosides $\mathrm{Rh} 2$ and $\mathrm{Rg} 3$ were the only degradation product of ginsenoside $\mathrm{Rc}$ and $\mathrm{Rd}$, respectively. The yields of ginsenoside $\mathrm{Rg} 3, \mathrm{Rh} 2$ and aglycone are the highest under $165^{\circ} \mathrm{C}$ and $15 \mathrm{~min}$. The highest transformation rate of total ginsenosides to ginsenoside $\operatorname{Rg} 3$ was $47.98 \%$ in neutral solution. And the conversion rate of major ginsenosides to aglycon was $78.11 \%$ in alkaline solution. Moreover, the yield of ginsenoside $\mathrm{Rg} 3$ obtained by microwave is $7.69 \mathrm{mg} / \mathrm{g}$ and 250 times as high as that obtained from red ginseng. The mechanism of saponins conversion may be related to hydrolyze glycosidic bond at C-20 and C-3 [25]. In addition, the degradation of ginsenosides may follow a first-order reaction in ethanol solution. And neutral ginsenosides are more stable than malonyl ginsenosides. The values of the rate constants of ginsenosides degradation were similar at the same temperature in both aqueous and 50\% ethanol-water extracts between the microwave and conventional heating methods. So microwave could replace conventional heating methods to apply on the extraction of ginsenosides [26].

\section{Chemical Methods}

3.1. Acid Hydrolysis. As we all know, ginsenosides can be decomposed to corresponding prosapogenins under acidic condition. Major ginsenosides could produce different rare ginsenosides under different acidic conditions. Ginsenosides $\mathrm{Rg} 1$ and Re were treated with $0.1 \mathrm{~N}-\mathrm{HCL}$ at $37^{\circ} \mathrm{C}$ for $120 \mathrm{~min}$. $\mathrm{N}-\mathrm{BuOH}$ extracts of the mixtures were applied to silica gel column chromatography to get the prosapogenin of Rgl and Re. Rgl-prosapogenin I and Re-prosapogenin I were not detected because they were decomposed to other complicated mixtures [27]. What is more, the $\mathrm{pH}$ is a determinant factor in the transformation process of ginsenosides. Ginsenosides could not be transformed under the neutral condition and would produce by-products under strong acid. Different concentrations of formic acid $(0.01 \%, 0.1 \%, 0.5 \%, 2 \%$, and $5 \%)$ were applied to the conversion of some sapiens. The results showed that $0.01 \%$ of formic acid provided the highest yields. Ginsenosides Rh1, Rh2, and Rg3, with $0.01 \%$ formic acid at $120^{\circ} \mathrm{C}$ for $4 \mathrm{~h}$, could transform into ginsenosides $\mathrm{Rk} 3$ and Rh4, Rk1 and Rg5, and Rk2 and Rh3, respectively. Besides, the total transformation efficiency was more than 20\% through formic acid-treating. And the cytotoxic effect of ginsenosides on human cancer cells is inversely related to the sugar number and sugar linkages rank as C-3 > C-6 > C-20 [28]. 20(S)Protopanaxatriol- (PPT-) type ginsenosides standards $\mathrm{Re}$, $\mathrm{Rg} 2$, and $\mathrm{Rf}$ were dissolved by $50 \%$ (v/v) methanol/water and the liquor $\mathrm{PH}$ was adjusted to 2.0 with formic acid. The liquors of saponins were heated to reflux $\left(60^{\circ} \mathrm{C}\right)$ for $120 \mathrm{mins}$. The result demonstrated that $20(\mathrm{~S})$ - Re could produce eight compounds; they were 20(S)-Rf2, 20(S)-Rg2, 20(R)-Rg2, 20(S)-Rh1, 20(R)-Rh1, F1, Rg6, and Rg4. 20(S)-Rg2 produced five compounds; they were 20(S)-Rf2, 20(S)-Rh1, 20(R)-Rh1, $\mathrm{Rg} 6$, and $\mathrm{Rg} 4$. And 20(S)-Rf produced five compounds; they were 20(S)-Rh1, 20(R)-Rh1, 20(S)-Rf3, Rg8, and Rg9. The transformation mechanisms of 20(S)-protopanaxatriol(PPT-) type ginsenosides include hydrolysis of saccharide substitution, $\Delta 20(21)$ or $\Delta 20(22)$ dehydration, and hydration addition reactions at $\mathrm{C}-24$ and $\mathrm{C}-25$. The chemical transformation pathways of 20(S)-PPT ginsenosides Re, Rg2, and Rf were showed in Figure 2 [29]. Beside formic acid, these acids can also be used in chemical transformation of ginsenosides, such as acetic acid, citric acid, lactic acid, tartaric acid, and HCL. Protopanaxadiol (PPD) ginsenosides could transform into Rg3 and $\Delta^{20}$-ginsenoside Rg3 under acid conditions. The yield of $\mathrm{Rg} 3$ was increased by increasing incubation temperature and time in acidic condition. The optimal time and temperature of transformation were $5 \mathrm{~h}$ and $60^{\circ} \mathrm{C}$, respectively. What is more, ginsenosides $\mathrm{Rb} 1, \mathrm{Rb} 2$, and $\mathrm{Rc}$ were incubation at $60^{\circ} \mathrm{C}$ in acetic acid, citric acid, lactic acid, tartaric acid, and HCL and the levels of transformed ginsenosides were measured. Compared with lactic acid and citric acid, HCL could increase the yield of 20(R)-ginsenoside $\operatorname{Rg} 3$ and $\Delta^{20}$-ginsenoside $\operatorname{Rg} 3$ to $20(\mathrm{~S})$-ginsenoside $\mathrm{Rg} 3$. The transformation of ginsenosides by $0.1 \%$ acids was better than that of $1 \%$ acids. But the transformation of ginsenosides to $\Delta^{20}$-ginsenoside $\operatorname{Rg} 3$ by $0.1 \%$ acid was not greater than that by $1 \%$ acids. The results demonstrated that ginsenosides were easily transformed in acidic conditions. However, it is important to control the $\mathrm{PH}$, time, and temperature for the conversion of ginsenosides in acid condition [30].

3.2. Alkaline Hydrolysis. Alkaline hydrolysis is a method to decompose saponins on the condition of high temperature, high pressure, and alkaline to obtain secondary saponins. But alkaline hydrolysis also has a strict requirement on the reaction temperature, pressure, and $\mathrm{PH}$. Total saponins would be hydrolyzed in boiling water bath with $2 \mathrm{~mol} / \mathrm{L} \mathrm{NaOH}$ aqueous solution for $8 \mathrm{~h}$ and extracted by EtoAc. It is interesting to note that a new compound, 20(R)-ginsenoside Rh19, could be isolated and identified by NMR and MS spectra analyses [31]. For alkaline hydrolysis, temperature and $\mathrm{PH}$ are two important conditions. In general, at the same time, the higher the $\mathrm{PH}$, the faster the hydrolysis. The hydrolysis rate of ginsenoside Ro was also related to temperature and $\mathrm{PH}$. The hydrolysis rate changes more obviously on the condition of $\mathrm{PH} 13$ and $60^{\circ} \mathrm{C}$, mainly hydrolyzing the ester bond at C-28. The hydrolysate of ginsenoside Ro by alkali was zingibrosideR1 [32]. Moreover, the yield of protopanaxadiol was the highest by alkaline hydrolysis when the solvent is isoamyl alcohol, and hydrolysis time is at $24 \mathrm{~h}$, normal pressure. But the yield of protopanaxadiol would be decreased when the heating time is more than $32 \mathrm{~h}$ in isoamyl alcohol. This showed that prolonged heating can lead to the degradation of saponins in high temperatures [33]. 


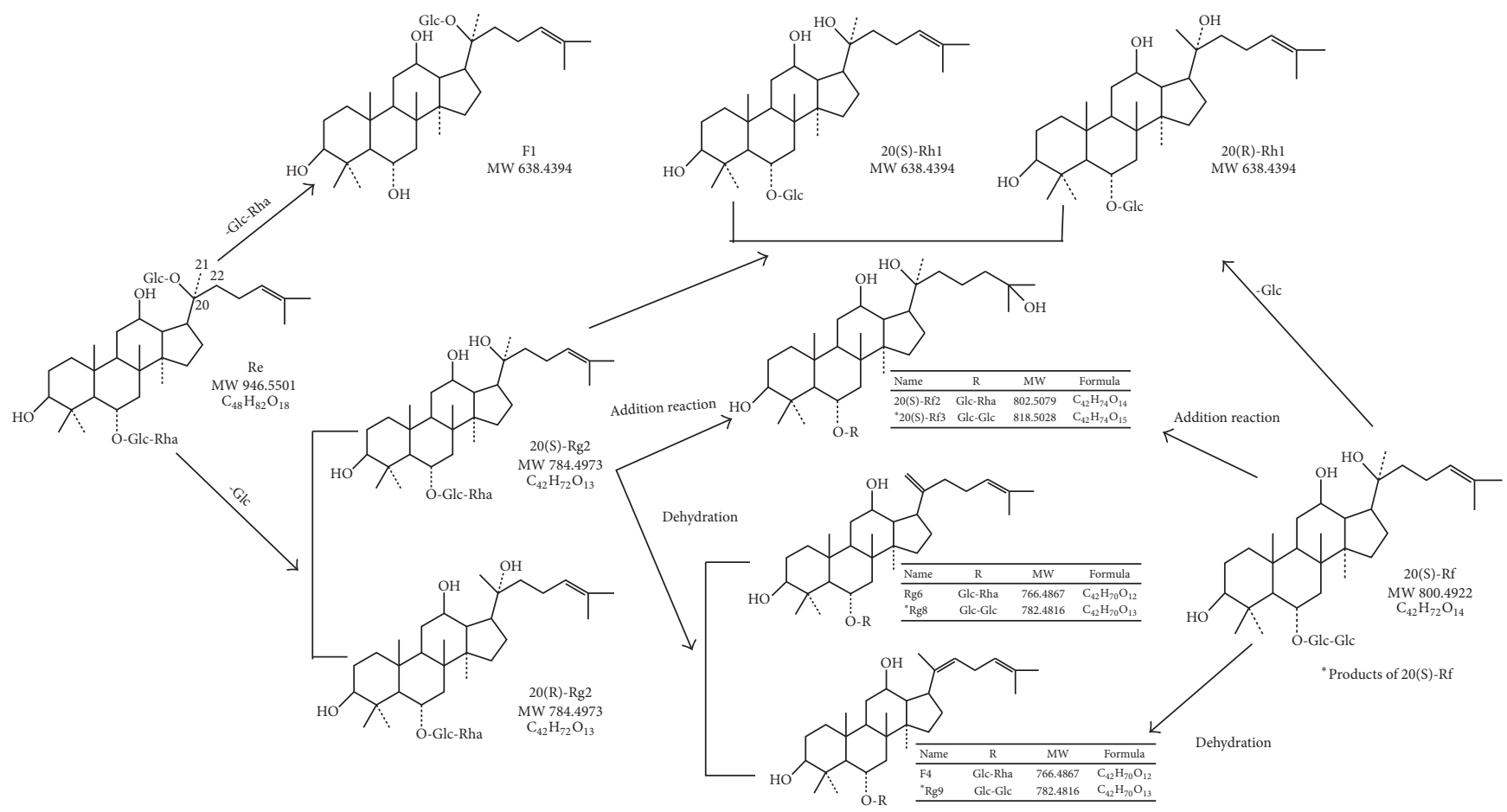

Figure 2: Data redrawn from [29]. Chemical transformation pathways of 20(S)-PPT ginsenosides Re, Rg2, and Rf. The transformation mechanisms of 20(S)-PPT ginsenosides Re, Rg2, and Rf include hydrolysis of saccharide substitution at C-6, $\Delta 20(21)$ or $\Delta 20(22)$ dehydration, and hydration addition reactions at C-24 and C-25.

These chemical methods also have some disadvantages, including poorly selective, epimerization, hydration, hydroxylation, and environmental pollution [34].

\section{Biotransformation of Ginsenosides}

Biotransformation is a way which makes use of organisms or enzyme as catalyst to realize the process of chemical conversion and modify the structure of the external substrate. The advantages of biotransformation are strongly selective, mildly reaction conditions, less by-products, simple reprocessing, friendly environment, and so on. The biotransformation of ginsenosides is mainly using microorganism or enzyme to decorate the glucosyl of ginsenosides at C-3, C-6, and C-20 to translate rare saponins. The main methods of biotransformation are enzyme conversion and microbial transformation.

4.1. Enzymatic Hydrolysis. The enzymatic way has been deemed to a promising method to generate rare ginsenosides, owing to its short reaction cycle, little pollution, high purity, and high specificity [34]. Different enzymes play a different role in hydrolyzing ginsenosides. Rapidase, Econase CE, Viscozyme, Ultraflo L, and Cytolase PC15 were used for secondary enzymatic hydrolysis after amylase treatment of ginseng extract. The hydrolysis reaction was stopped by boiling for $15 \mathrm{~min}$. The results showed that Rapidase can not only significantly increase the total contents of ginsenosides, but also increase the contents of panaxadiols and panaxatriols, such as Rh1, Rg5, Rk1, Rg2, and Rg3. However, the effect of Ultraflo L is opposite to Rapidase. Rc was the most ample in the control, but the contents of Rc are decreased after being treated with various enzymes. Some researches indicated that the hydrolysis of carbohydrates enhances the extraction of ginsenosides and shorter sugars [35]. Some commercial enzymes, Cytolase PCL5, Sumizyme AC, Multifect Pectinase FE, and Crystalzyme PML-MX, were added to the ginseng extract, mainly including ginsenosides Rb1, Rc, Rb2, and Rd. After $60 \mathrm{~h}$ of treatment by enzyme, these major ginsenosides were transformed into Rg3, F2 and compound K. what is more, the effects of Cytolase PCL5 and Sumizyme AC are better than other enzymes at conversion of ginsenosides. And Cytolase PCL5 is superior to Sumizyme AC because the yield of compound $\mathrm{K}$ is increased as the temperature decreased. The optimal conditions were identified as $78 \mathrm{~h}$ of treatment at $\mathrm{PH} 4.3$ at $55.4^{\circ} \mathrm{C}$. This result could provide a practical basis for other methods to hydrolyze saponins [36]. As is well known, ginsenoside $\mathrm{Rg} 3$ has the good effect on anticardiovascular and antimetastasis. But ginsenoside Rg3 is not found in the white ginseng and the content of it is very low in red ginseng. The 20(S)-Rg3 isomer is more water-soluble and more bioavailable than the $20(\mathrm{R})-\mathrm{Rg} 3$ isomer. There are few reports about the conversion of ginsenoside $\mathrm{Rg} 3$ by the enzymes. So it is important to explore the optimal condition for the transformation of ginsenoside $\mathrm{Rg} 3$ by commercial enzymes. Some commercial enzymes, Cellulase-12T, Protease NP, Viscozyme L, Celluclast 1.5L FG, Lactozym, Pectinex 5XL, Novozym 435, and Cytolase, were added to the white ginseng extract. The results indicated that the hydrolysis of Cellulase$12 \mathrm{~T}$ was more effective than other enzymes, by hydrolyzing the $\beta$-glycosidic linkage at C-20 in protopanaxadiol-type 
ginsenosides. Besides, the content of 20(S)-ginsenoside Rg3 could improve 4 times after treated with Cellulase-12T for $72 \mathrm{~h}$, comparing with commercial white ginseng extract [37]. It is known that aglycone protopanaxatriol is easy to be absorbed and has broader prospect than other saponins. Aglycone protopanaxatriol can be obtained by hydrolyzing the saponins. A recombinant $\beta$-glycosidase obtained from Dictyoglomus turgidum specifically hydrolyzed the xylose at the C-6 position and the glucose in protopanaxatriol- (PPT-) type ginsenosides. The specific activity of D. turgidum $\beta$ glycosidase followed the order: $\mathrm{Rf}>\mathrm{Rg} 1>\mathrm{Re}>\mathrm{R} 1>\mathrm{Rh} 1$ $>$ R2. But $D$. turgidum $\beta$-glycosidase did not have effect on $\operatorname{Rg} 2$. So $D$. turgidum $\beta$-glycosidase hydrolyzed the $\beta$-Dglucopyranose at the C-6 position and C-20 position and cannot hydrolyze $\alpha$-L-rhamnopyranoside at the C-6 position of $\mathrm{Rg} 2$. The optimal reaction conditions for APPT production using $D$. turgidum $\beta$-glycosidase were $\mathrm{pH} 6.0,80^{\circ} \mathrm{C}$. The transformed pathways of PPT-type ginsenosides using $D$. turgidum $\beta$-glycosidase were as follows: $\mathrm{R} 1 \rightarrow \mathrm{R} 2 \rightarrow \mathrm{Rh} 2 \rightarrow$ $\mathrm{APPT}, \mathrm{Rg} 1 \rightarrow \mathrm{Rh} 1 \rightarrow \mathrm{APPT}, \mathrm{Rf} \rightarrow \mathrm{Rh} 1 \rightarrow \mathrm{APPT}, \mathrm{Re} \rightarrow \mathrm{Rg} 2$ [38].

4.2. Intestinal Bacterial Hydrolysis. Many researchers study the metabolism of ginsenosides in vivo by intestinal microflora and found that the real effective component of ginsenosides is aglycone, which laid the foundation to the development of new drugs. The study found that 20-O- $\beta$-D-glucopyranosyl-20(S)-protopanaxadiol (IBM I), the intestinal bacterial metabolite of $\mathrm{Rb} 1$, was really resistant to metastasis instead of Rbl. And the antimetastatic efficacy of IBM I was better than that of Rb1 and at least comparable to that of 5-FU. And the cure rate of IBM I was greater than amputation alone in a leg amputation [39]. Besides, nine metabolites (M1-M9) of ginsenoside Rb1 were detected in rat feces and eight metabolites (M1, M2, M4-M8, and M10) were detected in rat urine. The structures of ten metabolites were identified as ginsenoside $\mathrm{Rd}$, gypenoside XVII, 20(S)-ginsenoside Rg3, 20(R)-ginsenoside Rg3, ginsenoside $\mathrm{F} 2$, compound $\mathrm{K}$, 12-hydroxydammar-3-one20(S)-O- $\beta$-D-glucopyranoside, and 25-hydroxyl-(E)-20(22)ene-ginsenoside $\mathrm{Rg} 3$, respectively. It is significant to know that four metabolites (M7-M10) were first reported in vivo. And the highest concentration of deglycosylated metabolites in rat urine and feces appeared during 6-12 h and $24-36 \mathrm{~h}$, respectively [40]. What is more, the same hydrolysates were found in human plasma and urine after human take oral extract but were not found in the extract. This result suggested that products must result from either the degradation or metabolism of ginsenosides in the human body. The major hydrolysates were ginsenoside Rh1, ginsenoside F1, and compound K. And compound K was the main metabolite of the protopanaxadiol ginsenosides [41]. The interesting thing is when ginsenoside Rbl was given orally to germ-free rat, neither compound $\mathrm{K}$ nor any other metabolite was detected in the plasma, intestinal tract, or cumulative feces. In contrast, when ginsenoside $\mathrm{Rbl}$ was given orally to gnotobiote rats monoassociated with Eubacterium sp. A-44, compound $\mathrm{K}$ was found in the plasma, caecum, and cumulative feces. The result indicated that ginsenoside $\mathrm{Rbl}$ was not easily absorbed into body. Nevertheless, compound $K$, the metabolic of ginsenoside Rb1, was easily absorbed into body. However, not all human intestinal could transform the ginsenoside Rb1. Eubacterium sp. A-44 is the only one intestinal strain from man that can convert ginsenoside $\mathrm{Rbl}$ into compound $\mathrm{K}$ via ginsenoside $\mathrm{Rd}$. The transformation pathway of ginsenoside $\mathrm{Rbl}$ by intestinal bacterial was as followed: $\mathrm{Rbl} \rightarrow \mathrm{Rd} \rightarrow \mathrm{F} 2 \rightarrow \mathrm{C}-\mathrm{K}$ $\rightarrow$ 20(S)-protopanaxadiol [42]. Furthermore, saponins can also be converted into metabolites in anaerobic environment. The possible transform pathways of ginsenosides Rb1, Rb2, $\mathrm{Rc}$, Re, and Rg1 were as follows: $\mathrm{Rb} 1 \rightarrow \mathrm{Rd} \rightarrow \mathrm{F} 2 \rightarrow 20$ O- $\beta$-D-glucopyranosyl-20(S)-protopanaxadiol, $\mathrm{Rb} 2 \rightarrow$ unknown-substance $\rightarrow$ 20-O-[ $\alpha$-L-arabinopyranosyl $(1 \rightarrow$ 6)- $\beta$-D-glucopyranosyl]-20(S)-protopanaxadiol $\rightarrow 20-\mathrm{O}-\beta$ D-glucopyranosyl-20(S)-protopanaxadiol, $\mathrm{Rc} \rightarrow \mathrm{Rh} 1 \rightarrow 20$ $\mathrm{O}$ - $[\alpha$-L-arabinofuranosyl $(1 \rightarrow 6)-\beta$-D-glucopyranosyl $]$ 20(S)-protopanaxadiol $\rightarrow$ 20-O- $\beta$-D-glucopyranosyl-20(S)protopanaxadiol, $\mathrm{Re} \rightarrow \mathrm{Rg} 1 \rightarrow \mathrm{F} 1 \rightarrow 20(\mathrm{~S})$-protopanaxatriol, $\mathrm{Rg} 1 \rightarrow \mathrm{F} 1 \rightarrow 20(\mathrm{~S})$-protopanaxatriol [43]. In addition, some human intestinal bacteria could transform ginsenoside Rg3 and $\triangle 20$-ginsenoside Rg3 to ginsenoside Rh2 and $\triangle 20$-ginsenoside $\mathrm{Rh} 2$ and protopanaxadiol. For example, Bacteroides sp., Eubacterium sp., and Bifidobacterium sp. could convert ginsenoside $\mathrm{Rg} 3$ to protopanaxadiol via ginsenoside Rh2, but Fusobacterium sp. metabolized ginsenoside $\operatorname{Rg} 3$ to ginsenoside $\mathrm{Rh} 2$ alone [30, 44]. The possible transformation pathway of $\operatorname{Rg} 3$ by fusobacterium $\mathrm{K}-60$ was as follows: ginsenoside $\mathrm{Rg} 3 \rightarrow$ ginsenoside $\mathrm{Rh} 2$ $\rightarrow$ protopanaxadiol, Rg3 $\rightarrow \triangle 20$-ginsenoside Rh2 $\rightarrow \triangle 20$ protopanaxadiol [44]. Another interesting thing is that 20O- $\beta$-D-glucopyranosyl-20(S)-protopanaxadiol, a metabolite of ginsenosides, was accumulated into the liver soon after its intravenous administration to mice and its metabolite was fatty acid ester that accumulated in the liver longer than it. Fatty acid ester inhibited tumor growth more than 20-O$\beta$-D-glucopyranosyl-20(S)-protopanaxadiol in vivo, which suggested that the real active substance of ginsenosides in the body may be fatty acid ester [30, 45].

4.3. Endophytic Bacteria Transformation. Endophyte is the fungus or bacterium that lives in the tissues and organs of healthy plants at a certain stage or all stage. $10 \beta$-glucosidaseproducing endophytic bacteria were isolated from ginseng roots. Among these endophytic bacteria, Burkholderia sp. GE 17-7 has a strong ability to convert the major ginsenoside $\mathrm{Rb} 1$ and $\mathrm{Rd}$ into minor ginsenoside $\mathrm{Rg} 3$ via hydrolyzing the outer glycosidic linkage at C-20 position. The pathway of ginsenoside Rb1 by the strain GE 17-7 was as follows: ginsenoside $\mathrm{Rb} \rightarrow$ ginsenoside $\mathrm{Rd} \rightarrow$ ginsenoside $\mathrm{Rg} 3$. The optimal conditions were $\mathrm{pH} 7.0,30^{\circ} \mathrm{C}, 15 \mathrm{~h}$ and the maximum conversion rate of ginsenoside $\mathrm{Rg} 3$ was $98 \%$ [46]. Ginsenosides $\mathrm{Rb} 1$ and $\mathrm{Rd}$ were also transformed into gypenoside LXXV and compound K by strain CNU 120806, respectively, by hydrolyzing the terminal and inner glucopyranosyl moieties at the C-3 carbon. The transformation pathways of ginsenosides Rbl and Rd were as follows: ginsenoside Rb1 $\rightarrow$ gypenoside XVII $\rightarrow$ gypenoside LXXV; ginsenoside $\mathrm{Rd} \rightarrow \mathrm{F} 2$ $\rightarrow$ compound $\mathrm{K}$. The optimal conditions for transformation 
TABLE 1: Ginsenosides converted by endophytic bacteria.

\begin{tabular}{lccc}
\hline Ginsenosides & Microorganism & After the transformation & References \\
\hline $\mathrm{Rb} 1$ & Burkholderia sp. GE 17-7 & $\operatorname{Rg} 3$ & {$[46]$} \\
& Esteya vermicola CNU 120806 & gypenoside LXXV & [47] \\
$\mathrm{Rb} 1, \mathrm{Rb} 2, \mathrm{Rc}, \mathrm{Rd}$ & Luteibacter $\mathrm{sp}$. & $\mathrm{F} 2, \mathrm{C}-\mathrm{K}$ & [49] \\
$\mathrm{Rg} 1$ & Luteibacter $\mathrm{sp}$. & $\mathrm{Rh} 1$ & {$[49]$} \\
$\mathrm{Rg} 3$ & Esteya vermicola CNU 120806 & {$[48]$} \\
\hline
\end{tabular}

by strain CNU 120806 are $50^{\circ} \mathrm{C}$ and pH 5.0 [47]. Besides, Esteya vermicola CNU 120806 has the high specific activity in converting ginsenoside $\mathrm{Rg} 3$ to Rh2. However, ginsenoside Re was not converted by strain CNU 120806 . The production rate of $\mathrm{Rh} 2$ reached $90.7 \%$. The optimal condition was as follows: $50^{\circ} \mathrm{C}, \mathrm{pH} 5.0$, and substrate concentration of $3 \mathrm{mg} \cdot \mathrm{ml}^{-1}$ [48]. An endophyte JG09 isolated from Platycodon grandiflorum also has the ability for converting the ginseng total saponins and ginsenoside monomers. For example, endophyte bacteria JG09 could transform ginsenosides Rb1, Rb2, Rc, and Rd into ginsenosides $\mathrm{F} 2$ and compound $\mathrm{K}$ and transformed ginsenoside Rgl into ginsenoside Rhl. The probable pathways of Rb1, Rb2, Rc, and Rg1 by endophyte JG09 were as follows: $\mathrm{Rb} 1 \rightarrow \mathrm{Rd} \rightarrow \mathrm{F} 2 \rightarrow \mathrm{C}-\mathrm{K} ; \mathrm{Rb} 2 \rightarrow \mathrm{C}-\mathrm{O} \rightarrow \mathrm{C}-\mathrm{Y} \rightarrow \mathrm{C}-\mathrm{K} ; \mathrm{Rc}$ $\rightarrow \mathrm{C}-\mathrm{Mcl} \rightarrow \mathrm{C}-\mathrm{Mc} \rightarrow \mathrm{C}-\mathrm{K} ; \mathrm{Rg} 1 \rightarrow \mathrm{Rh} 1$. (Table 1). And the maximum production rate of ginsenoside $\mathrm{F} 2$ and compound $\mathrm{K}$ reached $94.53 \%$ and $66.34 \%$ on the condition of $\mathrm{PH} 4.0$, respectively [49].

4.4. Edible Fungi Transformation. Microorganisms applied on the transformation of ginsenoside do not have the foodgrade standards. But on the influence of food safety, microorganisms isolated from food have a great prospect at industrial production. Leuconostoc mesenteroides DC102 strain and Lactobacillus pentosus strain 6105 were isolated from kimchi, a traditional Korean fermented food. Leuconostoc mesenteroides DC102 would convert ginsenoside Rb1 to gypenoside XVII, ginsenoside $\mathrm{Rd}$, ginsenoside $\mathrm{F} 2$, and compound $\mathrm{K}$. Leuconostoc mesenteroides DC102 converted ginsenoside Rb1 to compound $\mathrm{K}$ by hydrolyzing the two glucose molecules at C-3 and one of the glucose molecules at C-20, suggesting the conversion pathway: ginsenoside $\mathrm{Rb} 1 \rightarrow$ gypenoside XVII and ginsenoside $\mathrm{Rd} \rightarrow$ ginsenoside $\mathrm{F} 2 \rightarrow$ compound $\mathrm{K}$. The optimal conditions of conversion were as follows: $30^{\circ} \mathrm{C}, 72 \mathrm{~h}$ and PH 6.0 to 8.0. [50, 51]. Lactobacillus paralimentarius LH4 is also isolated from kimchi and it transformed ginsenoside Rb1 into compound $\mathrm{K}$ via gypenoside XVII, ginsenoside $\mathrm{Rd}$, and ginsenoside F2 orderly, through hydrolyzing the two glucose moieties at C-3 and the outer glucose moiety at $\mathrm{C}-20$ position of the ginsenoside Rbl. And the optimal conditions of conversion by Lactobacillus paralimentarius $\mathrm{LH} 4$ were $30^{\circ} \mathrm{C}, \mathrm{pH} 6.0$, and $72 \mathrm{~h}$ [52]. In addition, some food microorganisms could convert ginsenoside $\mathrm{Rb} 1$ and $\mathrm{Re}$ into rare ginsenosides. The conversion pathway of ginsenoside $\mathrm{Rb} 1$ and Re by food microorganisms can be traced as follows: $\mathrm{Rb} 1 \rightarrow \mathrm{Rd}$ and $\mathrm{F} \rightarrow$ compound $\mathrm{K}, \mathrm{Rb} 1 \rightarrow \mathrm{Rd}$ and $\mathrm{F} 2 \rightarrow$ $\mathrm{Rh} 2, \mathrm{Rb} 1 \rightarrow \mathrm{Rd} \rightarrow \mathrm{F} 2, \mathrm{Re} \rightarrow \mathrm{Rg} 2 \rightarrow \mathrm{Rh} 1, \mathrm{Re} \rightarrow \mathrm{Rg} 1 \rightarrow \mathrm{Rh} 1$ [53]. Ginsenosides Rb2 and Rc were also transformed by food microorganisms. The conversion pathways of ginsenosides
$\mathrm{Rb} 2$ and Rc were as follows: $\mathrm{Rb} 2$ and $\mathrm{Rc} \rightarrow \mathrm{Rd} \rightarrow \mathrm{F} 2 \rightarrow$ $\mathrm{Rh} 2$ or compound $\mathrm{K}, \mathrm{Rb} 2 \rightarrow$ compound $\mathrm{O} \rightarrow$ compound $\mathrm{Y}$ $\rightarrow$ compound $\mathrm{K}, \mathrm{Rc} \rightarrow$ compound $\mathrm{Mc} \rightarrow$ compound $\mathrm{K}$ [54] (Table 2).

4.5. Soil Microbial Transformation. Soil microbes are the microscopic organisms that are invisible to the naked eye or invisible to the soil. Strictly speaking, they include bacteria, archaea, fungi, viruses, protozoa, and microscopic algae. They can make chemical reactions in the soil and promote the decomposition of soil organic matter and the transformation of nutrients. Twenty-two microorganisms, producing $\beta$ glucosidase, were isolated from the ginseng filed. Among these microorganisms, GH9, GH21, and GH26 have the ability to transform ginsenoside Rb1 to less polar metabolite. Only GH21 was able to transform ginsenoside Rg1. GH21 was identified as a Cladosporium cladosporioides species by comparing rRNA gene sequences. The proposed conversion pathways of ginsenosides Rb1 and Rg1 by GH21 were as follows: $\mathrm{Rb} 1 \rightarrow \mathrm{Rd}$ or gypenoside $\mathrm{XVII} \rightarrow \mathrm{F} 2 \rightarrow \mathrm{C}-\mathrm{K}, \mathrm{Rg} 1$ $\rightarrow$ F1, respectively. Ginsenosides Rb1 and Rg1 bioconversion rates were $74.2 \%$ and $89.3 \%$, respectively [55]. Aspergillus versicolor strain LFJ1403, producing $\beta$-glucosidase, is also isolated from ginseng filed. Strain LFJ1403 could transform ginsenoside $\mathrm{Rb} 1$ into ginsenoside $\mathrm{Rd}$ as the sole product by hydrolyzing the outer glucose moiety at C-20 position. The yield of conversion was up to $96 \%$ on the optimal biotransformation conditions, $37^{\circ} \mathrm{C}, \mathrm{PH} 5.0$, and $48 \mathrm{~h}$ [56]. In addition, many microorganisms were obtained around the ginseng roots in fields. Among these microorganisms, a strain of fungus GF06 was identified as Aspergillus niger and showed a strong ability to transform $\operatorname{Rg} 3(\mathrm{~S}, \mathrm{R})$ into $\mathrm{PPD}(\mathrm{S}, \mathrm{R})$ via the following pathway: $\mathrm{Rg} 3 \rightarrow \mathrm{Rh} 2 \rightarrow \mathrm{PPD}, \mathrm{Rg} 3 \rightarrow \mathrm{PPD}$. The conversion rate of $\mathrm{Rg} 3(\mathrm{~S}, \mathrm{R})$ into $\mathrm{PPD}(\mathrm{S}, \mathrm{R})$ reached $100 \%$, and the optimal conditions were PH5.0 and $55^{\circ} \mathrm{C}$ [57]. What is more, Aspergillus niger also could transform ginsenoside $\mathrm{Rf}$ to 20 (S)-protopanaxatriol by hydrolyzing the terminal glucose and then inner glucose at the C-6 position, suggesting the transformation pathway: $\mathrm{Rf} \rightarrow \mathrm{Rh1}(\mathrm{S}) \rightarrow \mathrm{PPT}(\mathrm{S})$. The yield of conversion was up to $90.4 \%$ on the optimal conditions, $\mathrm{PH} 5.0,55^{\circ} \mathrm{C}$ and substrate concentration $1.25 \mathrm{mmol} / \mathrm{l}$ [58]. Beside, Microbacterium esteraromaticum would convert ginsenoside Rb2 into compound $\mathrm{Y}$ and compound $\mathrm{K}$. The possible conversion mechanism was as follows: $\mathrm{Rb} 2 \rightarrow$ compound Y $\rightarrow$ compound K [59]. Moreover, Fusarium sacchari, isolated from the soil-cultivated ginseng, could transform leaves of total saponins into compound $\mathrm{K}$, compound $\mathrm{Mx}$, and ginsenoside $\mathrm{Mc}$, which have the strong pharmacological activity. And the levels of $\mathrm{C}-\mathrm{K}, \mathrm{C}-\mathrm{Mx}$, and $\mathrm{G}-\mathrm{Mc}$ were 
TABLE 2: Ginsenosides converted by edible fungi.

\begin{tabular}{|c|c|c|c|}
\hline Ginsenosides & Microorganism & After the transformation & References \\
\hline \multirow{7}{*}{$\mathrm{Rb} 1$} & Leuconostoc mesenteroides DC102 & $\begin{array}{l}\text { Prosapogenins, Rd, F2, } \\
\text { gypenoside XVII, C- K }\end{array}$ & {$[51]$} \\
\hline & Lactobacillus pentosus strain 6105 & PPD & {$[52]$} \\
\hline & Lactobacillus paralimentarius, & & \\
\hline & $\begin{array}{c}\text { Bifidobacterium sp. Int57, Bif. sp. SJ32, Aspergillus } \\
\text { niger and A. usamii }\end{array}$ & $\mathrm{C}-\mathrm{K}$ & {$[53,54]$} \\
\hline & Lactobacillus delbrueckii, & Rh2 & {$[54]$} \\
\hline & Leuconostoc paramesenteroides & & \\
\hline & Bifidobacterium sp. SH5 & $\mathrm{F} 2$ & {$[54]$} \\
\hline \multirow{2}{*}{$\operatorname{Re}$} & Bif. sp. Int57, Bif. sp. SJ32, A. niger & Rh1 & {$[54]$} \\
\hline & A. usamii & $\operatorname{Rg} 2$ & {$[54]$} \\
\hline \multirow{4}{*}{$\mathrm{Rb} 2, \mathrm{Rc}$} & Bifidobacterium sp. Int57, & & \\
\hline & Bifidobacterium sp. SJ32, & $\mathrm{C}-\mathrm{K}$ & {$[55]$} \\
\hline & Bifidobacterium sp. SH5 & & \\
\hline & Lactobacillus delbrueckii & Rh2 & [55] \\
\hline
\end{tabular}

TABLE 3: Ginsenosides converted by soil microbacteria.

\begin{tabular}{lccc}
\hline Ginsenosides & Microorganism & After the transformation & References \\
\hline $\mathrm{Rb} 1, \mathrm{Rg} 1$ & Cladosporium cladosporioides & $\mathrm{C}-\mathrm{K}, \mathrm{F} 1$ & $\mathrm{Rd}$ \\
$\mathrm{Rb} 1$ & Aspergillus versicolor & $\mathrm{C}-\mathrm{Y}, \mathrm{C}-\mathrm{K}$ & {$[56]$} \\
$\mathrm{Rb} 2$ & Microbacterium esteraromaticum & $\mathrm{PPD}(\mathrm{S}, \mathrm{R})$ & {$[57]$} \\
$\mathrm{Rg} 3(\mathrm{~S}, \mathrm{R})$ & Aspergillus niger & 20(S)-protopanaxatriol & {$[60]$} \\
$\mathrm{Rf}$ & Aspergillus niger & $\mathrm{C}-\mathrm{K}, \mathrm{C}-\mathrm{Mx}, \mathrm{G}-\mathrm{Mc}$ & {$[60]$} \\
$\mathrm{Rb} 1, \mathrm{Rb} 2, \mathrm{Rb} 3, \mathrm{Rc}$ & Fusarium sacchari & {$[61]$} \\
\hline
\end{tabular}

the highest in the PDA medium, comparing with martin, wort media, and Zpek medium. The optimal transforming conditions for C-K, C-Mx, and G-Mc by Fusarium sacchari were as follows: $30^{\circ} \mathrm{C}, 6$ days, and PH 5.5. The content of CK converted by Fusarium sacchari was increased by 215 times those of the control [60] (Table 3).

\section{Conclusion}

In conclusion, the transformation of ginsenosides is achieved mainly by hydrolyzing the glycosidic bond at C-3, C-6, and C20 position of the ginsenosides. Transformation of saponins by physical methods is not suitable for industrial production because of the high reaction conditions, the huge energy consumption, and the lower level of saponins. For instant, the content of PPD and PPT would be decreased at steaming. The secondary saponins prepared by chemical methods were easy to hydroxylate, hydrolyze, and isomerize. There are some problems in reaction progress, such as violent reaction, uncontrolled hydrolysis degree, difficult purification, and environmental pollution. Moreover, the transformation rate of secondary saponins was not high by chemical methods. Comparing the physical methods and chemical methods, biotransformation has quite a few advantages in the transformation of ginsenosides. For example, biotransformation is a fast, convenient, efficient, and environmental friendly way to obtain rare saponins. Because of these advantages, biotransformation is also the most commonly used method to convert ginsenoside. More significantly, China is the first to realize the industrial production of the Rh2 and other rare saponins by using enzymatic method [61]. In recent years, a large number of researches have been studied for the conversion of major ginsenosides and have achieved great results. However, these methods cannot meet the requirement of industrialization in producing rare ginsenosides. It needs more researchers to explore the way to produce the rare ginsenosides and be applied on large-scale production of saponins.

\section{Conflicts of Interest}

All contributing authors declare that there are no conflicts of interest.

\section{Acknowledgments}

This work was supported by Project of Shandong Province Key Research and Development Program (2017YYSP030).

\section{References}

[1] J.-H. Kim, "Cardiovascular diseases and panax ginseng: a review on molecular mechanisms and medical applications," Journal of Ginseng Research, vol. 36, no. 1, pp. 16-26, 2012. 
[2] Y. Sun, Y. Liu, and K. Chen, "Roles and mechanisms of ginsenoside in cardiovascular diseases: progress and perspectives," SCIENCE CHINA Life Sciences, vol. 59, no. 3, pp. 292-298, 2016.

[3] Q. Wang, H.-X. Li, L.-M. Liu, L.-M. Bi, S.-Y. Liu, and H.-F. Zhang, "The effect Panax ginseng tonic in the treatment of alzheimer's disease," Hebei Medical Journal, vol. 38, no. 22, pp. 3460-3465, 2016.

[4] J. Niu, Z. Pi, H. Yue, Y. Wang, Q. Yu, and S. Liu, "Effect of ginseng polysaccharide on the urinary excretion of type 2 diabetic rats studied by liquid chromatography-mass spectrometry," Journal of Chromatography B, vol. 907, pp. 7-12, 2012.

[5] V. A. Assinewe, J. T. Arnason, A. Aubry, J. Mullin, and I. Lemaire, "Extractable polysaccharides of Panax quinquefolius L. (North American ginseng) root stimulate TNF $\alpha$ production by alveolar macrophages," Phytomedicine, vol. 9, no. 5, pp. 398404, 2002.

[6] J. Wang, S. Li, Y. Fan et al., "Anti-fatigue activity of the watersoluble polysaccharides isolated from Panax ginseng C. A. Meyer," Journal of Ethnopharmacology, vol. 130, no. 2, pp. 421423, 2010.

[7] K.-C. Shin and D.-K. Oh, "Characterization of a novel recombinant $\beta$-glucosidase from Sphingopyxis alaskensis that specifically hydrolyzes the outer glucose at the C-3 position in protopanaxadiol-type ginsenosides," Journal of Biotechnology, vol. 172, no. 1, pp. 30-37, 2014.

[8] L. Wang, S.-J. Zhao, Y.-L. Liang, Y. Sun, H.-J. Cao, and Y. Han, "Identification of the protopanaxatriol synthase gene CYP6H for ginsenoside biosynthesis in Panax quinquefolius," Functional \& Integrative Genomics, vol. 14, no. 3, pp. 559-570, 2014.

[9] S. Han, A. J. Jeong, H. Yang et al., "Ginsenoside 20(S)$\mathrm{Rh} 2$ exerts anti-cancer activity through targeting IL-6-induced JAK2/STAT3 pathway in human colorectal cancer cells," Journal of Ethnopharmacology, vol. 194, pp. 83-90, 2016.

[10] I.-S. Lee, I. Uh, K.-S. Kim et al., "Anti-inflammatory effects of ginsenoside Rg3 via NF- $\kappa$ B pathway in A549 cells and human asthmatic lung tissue," Journal of Immunology Research, vol. 2016, Article ID 7521601, 2016.

[11] W. Hu, P. Jing, L. Wang, Y. Zhang, J. Yong, and Y. Wang, “The positive effects of Ginsenoside Rgl upon the hematopoietic microenvironment in a D-Galactose-induced aged rat model," BMC Complementary and Alternative Medicine, vol. 15, p. 119, 2015.

[12] X. Dong, L. Zheng, S. Lu, and Y. Yang, "Neuroprotective effects of pretreatment of ginsenoside Rbl on severe cerebral ischemiainduced injuries in aged mice: involvement of anti-oxidant signaling," Geriatrics \& Gerontology International, vol. 17, no. 2, pp. 338-345, 2017.

[13] E. A. Bae, M. J. Han, M. K. Choo, S. Y. Park, and D. H. Kim, "Metabolism of 20(S)- and 20(R)-ginsenoside Rg3 by human intestinal bacteria and its relation to in vitro biological activities," Biol Pharm Bull, vol. 25, no. 1, pp. 58-63, 2002.

[14] H.-M. Liu, D.-J. Chen, J. Yang, and X.-L. Piao, "Research progress on microbial transformation of saponin," Lishizhen Medicine And Materia Medica, vol. 24, no. 7, pp. 1722-1724, 2013.

[15] F. Zheng, W. Zhang, X. Chu et al., "Genome sequencing of strain Cellulosimicrobium sp. TH-20 with ginseng biotransformation ability," 3 Biotech, vol. 7, no. 4, 237 pages, 2017.

[16] C.-L. Guo, X.-M. Cui, X.-Y. Yang, and S. Wu, "Advances in studies on biotransformation of ginsensides," Zhongguo Zhong Yao Za Zhi, vol. 39, no. 20, pp. 3899-3904, 2014.
[17] Y.-Y. Xie, D. Luo, Y.-J. Cheng et al., "Steaming-induced chemical transformations and holistic quality assessment of red ginseng derived from Panax ginseng by means of HPLC-ESI-MS/MS ${ }^{n}$ based multicomponent quantification fingerprint," Journal of Agricultural and Food Chemistry, vol. 60, no. 33, pp. 8213-8224, 2012.

[18] W. Y. Kim, J. M. Kim, S. B. Han et al., "Steaming of ginseng at high temperature enhances biological activity," Journal of Natural Products, vol. 63, no. 12, pp. 1702-1704, 2000.

[19] Y.-S. Keum, K.-K. Park, and J.-M. Lee, "Antioxidant and antitumor promoting activities of the methanol extract of heatprocessed ginseng," Cancer Letters, vol. 150, no. 1, pp. 41-48, 2000.

[20] Z. Liu, J. Xia, C.-Z. Wang et al., "Remarkable Impact of Acidic Ginsenosides and Organic Acids on Ginsenoside Transformation from Fresh Ginseng to Red Ginseng," Journal of Agricultural and Food Chemistry, vol. 64, no. 26, pp. 5389-5399, 2016.

[21] L. Wang, X. Yang, X. Yu, Y. Yao, and G. Ren, "Evaluation of antibacterial and anti-inflammatory activities of less polar ginsenosides produced from polar ginsenosides by heattransformation," Journal of Agricultural and Food Chemistry, vol. 61, no. 50, pp. 12274-12282, 2013.

[22] X. Jin, L.-Y. Zhu, H. Shen et al., "Influence of sulphurfumigation on the quality of white ginseng: A quantitative evaluation of major ginsenosides by high performance liquid chromatography," Food Chemistry, vol. 135, no. 3, pp. 1141-1147, 2012.

[23] S.-L. Li, H. Shen, L.-Y. Zhu et al., "Ultra-high-performance liquid chromatography-quadrupole/time of flight mass spectrometry based chemical profiling approach to rapidly reveal chemical transformation of sulfur-fumigated medicinal herbs, a case study on white ginseng," Journal of Chromatography A, vol. 1231, pp. 31-45, 2012.

[24] H. Zhu, H. Shen, J. Xu et al., "Comparative study on intestinal metabolism and absorption in vivo of ginsenosides in sulphurfumigated and non-fumigated ginseng by ultra performance liquid chromatography quadruple time-of-flight mass spectrometry based chemical profiling approach," Drug Testing and Analysis, vol. 7, no. 4, pp. 320-330, 2015.

[25] Y. Bai, L. Zhao, C. Qu, X. Meng, and H. Zhang, "Microwave degradation of floatation-enriched ginsenoside extract from Panax quinquefolium L. Leaf," Journal of Agricultural and Food Chemistry, vol. 57, no. 21, pp. 10252-10260, 2009.

[26] G. Ren and F. Chen, "Degradation of ginsenosides in American ginseng (Panax quinquefolium) extracts during microwave and conventional heating," Journal of Agricultural and Food Chemistry, vol. 47, no. 4, pp. 1501-1505, 1999.

[27] B. H. Han, M. H. Park, and Y. N. Han, "Degradation of ginseng saponins under mild acidic conditions," Planta Medica, vol. 44, no. 3, pp. 146-149, 1982.

[28] K. Quan, Q. Liu, J.-Y. Wan et al., "Rapid preparation of rare ginsenosides by acid transformation and their structure-activity relationships against cancer cells," Scientific Reports, vol. 5, article no. 8598, 2015.

[29] W. Wu, Q. Qin, Y. Guo, J. Sun, and S. Liu, "Studies on the chemical transformation of 20(S)-protopanaxatriol (PPT)-type ginsenosides $\mathrm{Re}, \mathrm{Rg} 2$, and $\mathrm{Rf}$ using rapid resolution liquid chromatography coupled with quadruple-time-of-flight mass spectrometry (RRLC-Q-TOF-MS)," Journal of Agricultural and Food Chemistry, vol. 60, no. 40, pp. 10007-10014, 2012. 
[30] E.-A. Bae, J. H. Myung, E.-J. Kim, and D.-H. Kim, "Transformation of ginseng saponins to ginsenoside Rh2 by acids and human intestinal bacteria and biological activities of their transformants," Archives of Pharmacal Research, vol. 27, no. 1, pp. 61-67, 2004.

[31] L.-Y. Ma and X.-W. Yang, "20(R)-Ginsenoside-Rh19, a novel ginsenoside from alkaline hydrolysates of total saponins in stems-leaves of Panax ginseng," Chinese Traditional and Herbal Drugs, vol. 47, no. 1, pp. 6-14, 2016.

[32] Q. Zhu, D.-K. Li, D.-Z. Zhou, Z.-L. Ye, and W.-Y. Liu, "Study on hydrolysis kinetics of ginsenoside-Ro in alkaline medium and structural analysis of its hydrolyzate," Zhongguo Zhongyao Zazhi, vol. 39, no. 5, pp. 867-872, 2014.

[33] C. Zhang, F. Guan, and L. Zhang, "A preliminary study on the improvement of protopanaxadiol yield by alkaline hydrolysis," Journal of Baotou Medical College, vol. 27, no. 6, pp. 1-5, 2011.

[34] C.-S. Park, M.-H. Yoo, K.-H. Noh, and D.-K. Oh, "Biotransformation of ginsenosides by hydrolyzing the sugar moieties of ginsenosides using microbial glycosidases," Applied Microbiology and Biotechnology, vol. 87, no. 1, pp. 9-19, 2010.

[35] H.-S. Choi, S. Y. Kim, Y. Park, E. Y. Jung, and H. J. Suh, "Enzymatic transformation of ginsenosides in korean red ginseng (Panax ginseng meyer) extract prepared by spezyme and optidex," Journal of Ginseng Research, vol. 38, no. 4, pp. 264269, 2014

[36] E. H. Kim, S. Lim, S. O. Kim, S. H. Ahn, and Y. J. Choi, "Optimization of enzymatic treatment for compound $\mathrm{k}$ production from white ginseng extract by response surface methodology," Bioscience, Biotechnology, and Biochemistry, vol. 77, no. 5, pp. 1138-1140, 2013.

[37] K.-H. Chang, H. S. Jee, N.-K. Lee, S.-H. Park, N.-W. Lee, and H.D. Paik, "Optimization of the enzymatic production of 20(S)ginsenoside $\mathrm{Rg} 3$ from white ginseng extract using response surface methodology," New Biotechnology, vol. 26, no. 3-4, pp. 181-186, 2009.

[38] H.-J. Lee, K.-C. Shin, G.-W. Lee, and D.-K. Oh, "Production of aglycone protopanaxatriol from ginseng root extract using Dictyoglomus turgidum $\beta$-glycosidase that specifically hydrolyzes the xylose at the C-6 position and the glucose in protopanaxatriol-type ginsenosides," Applied Microbiology and Biotechnology, vol. 98, no. 8, pp. 3659-3667, 2014.

[39] H. Hasegawa and M. Uchiyama, "Antimetastatic efficacy of orally administered ginsenoside Rb1 in dependence on intestinal bacterial hydrolyzing potential and significance of treatment with an active bacterial metabolite," Planta Medica, vol. 64, no. 8, pp. 696-700, 1998.

[40] G. Chen, M. Yang, Y. Song et al., "Comparative analysis on microbial and rat metabolism of ginsenoside Rb1 by highperformance liquid chromatography coupled with tandem mass spectrometry," Biomedical Chromatography, vol. 22, no. 7, pp. 779-785, 2008.

[41] M. A. Tawab, U. Bahr, M. Karas, M. Wurglics, and M. SchubertZsilavecz, "Degradation of ginsenosides in humans after oral administration," Drug Metabolism and Disposition, vol. 31, no. 8, pp. 1065-1071, 2003.

[42] T. Akao, H. Kida, M. Kanaoka, M. Hattori, and K. Kobashi, "Intestinal bacterial hydrolysis is required for the appearance of compound $\mathrm{K}$ in rat plasma after oral administration of ginsenoside Rb1 from Panax ginseng," Journal of Pharmacy and Pharmacology, vol. 50, no. 10, pp. 1155-1160, 1998.
[43] H. Hasegawa, J.-H. Sung, S. Matsumiya, and M. Uchiyama, "Main ginseng saponin metabolites formed by intestinal bacteria," Planta Medica, vol. 62, no. 5, pp. 453-457, 1996.

[44] E. A. Bae, M. J. Han, M. K. Choo, and S. Y. Park, "Metabolism of 20(S)- and 20(R)-ginsenoside Rg3 by human intestinal bacteria and its relation to in vitro biological activities," Biol Pharm Bull, vol. 25, no. 1, pp. 58-63, 2002.

[45] H. Hasegawa, K.-S. Lee, T. Nagaoka et al., "Pharmacokinetics of ginsenoside deglycosylated by intestinal bacteria and its transformation to biologically active fatty acid esters," Biological \& Pharmaceutical Bulletin, vol. 23, no. 3, pp. 298-304, 2000.

[46] Y. Fu, Z. Yin, and C. Yin, " Biotransformation of ginsenoside $\mathrm{Rb} 1$ to ginsenoside Rg3 by endophytic bacterium ," Journal of Applied Microbiology, vol. 122, no. 6, pp. 1579-1585, 2017.

[47] J.-G. Hou, J.-J. Xue, M.-Q. Sun et al., "Highly selective microbial transformation of major ginsenoside Rb1 to gypenoside LXXV by Esteya vermicola CNU120806," Journal of Applied Microbiology, vol. 113, no. 4, pp. 807-814, 2012.

[48] J. Hou, J. Xue, C. Wang et al., "Microbial transformation of ginsenoside Rg3 to ginsenoside Rh2 by Esteya vermicola CNU 120806," World Journal of Microbiology and Biotechnology, vol. 28, no. 4, pp. 1807-1811, 2012.

[49] L. Cui, S.-Q. Wu, C.-A. Zhao, and C.-R. Yin, "Microbial conversion of major ginsenosides in ginseng total saponins by Platycodon grandiflorum endophytes," Journal of Ginseng Research, vol. 40, no. 4, pp. 366-374, 2016.

[50] L.-H. Quan, J.-Y. Piao, J.-W. Min et al., "Biotransformation of ginsenoside $\mathrm{Rb} 1$ to prosapogenins, gypenoside XVII, ginsenoside $\mathrm{Rd}$, ginsenoside $\mathrm{F} 2$, and compound $\mathrm{K}$ by leuconostoc mesenteroides DC102," Journal of Ginseng Research, vol. 35, no. 3, pp. 344-351, 2011.

[51] S.-H. Kim, J.-W. Min, L.-H. Quan, S. Lee, D.-U. Yang, and D.-C. Yang, "Enzymatic transformation of ginsenoside Rbl by lactobacillus pentosus strain 6105 from Kimchi," Journal of Ginseng Research, vol. 36, no. 3, pp. 291-297, 2012.

[52] L.-H. Quan, Y.-J. Kim, G. H. Li, K.-T. Choi, and D.-C. Yang, "Microbial transformation of ginsenoside $\mathrm{Rb} 1$ to compound $\mathrm{K}$ by Lactobacillus paralimentarius," World Journal of Microbiology and Biotechnology, vol. 29, no. 6, pp. 1001-1007, 2013.

[53] H. Chi and G.-E. Ji, "Transformation of ginsenosides Rbl and Re from Panax ginseng by food microorganisms," Biotechnology Letters, vol. 27, no. 11, pp. 765-771, 2005.

[54] H. Chi, D.-H. Kim, and G.-E. Ji, "Transformation of ginsenosides Rb2 and Rc from Panax ginseng by food microorganisms," Biological \& Pharmaceutical Bulletin, vol. 28, no. 11, pp. 21022105, 2005

[55] L. Wu, Y. Jin, C. Yin, and L. Bai, "Co-transformation of Panax major ginsenosides $\mathrm{Rbl}$ and $\mathrm{Rg} 1$ to minor ginsenosides $\mathrm{C}-\mathrm{K}$ and F1 by Cladosporium cladosporioides," Journal of Industrial Microbiology and Biotechnology, vol. 39, no. 4, pp. 521-527, 2012.

[56] F. Lin, X. Guo, and W. Lu, "Efficient biotransformation of ginsenoside $\mathrm{Rb} 1$ to $\mathrm{Rd}$ by isolated Aspergillus versicolor, excreting $\beta$-glucosidase in the spore production phase of solid culture," Antonie van Leeuwenhoek-Journal of Microbiology, vol. 108, no. 5, pp. 1117-1127, 2015.

[57] L. Liu, X.-M. Zhu, Q.-J. Wang et al., "Enzymatic preparation of 20(S, R)-protopanaxadiol by transformation of 20(S, R)-Rg3 from black ginseng," Phytochemistry, vol. 71, no. 13, pp. 15141520, 2010.

[58] L.-H. Quan, Y. Jin, C. Wang, J.-W. Min, Y.-J. Kim, and D.-C. Yang, "Enzymatic transformation of the major ginsenoside Rb2 
to minor compound $\mathrm{Y}$ and compound $\mathrm{K}$ by a ginsenosidehydrolyzing $\beta$-glycosidase from Microbacterium esteraromaticum," Journal of Industrial Microbiology and Biotechnology, vol. 39, no. 10, pp. 1557-1562, 2012.

[59] L. Liu, L.-J. Gu, D.-L. Zhang et al., "Microbial conversion of rare ginsenoside Rf to 20(S)-protopanaxatriol by Aspergillus niger," Bioscience, Biotechnology, and Biochemistry, vol. 74, no. 1, pp. 96-100, 2010.

[60] Y. Han, B. Sun, X. Hu et al., "Transformation of bioactive compounds by Fusarium sacchari fungus isolated from the soilcultivated ginseng," Journal of Agricultural and Food Chemistry, vol. 55, no. 23, pp. 9373-9379, 2007.

[61] X.-F. Wang, "Industrial production of ginsenosides," Health Daily, 2004-2-23. 

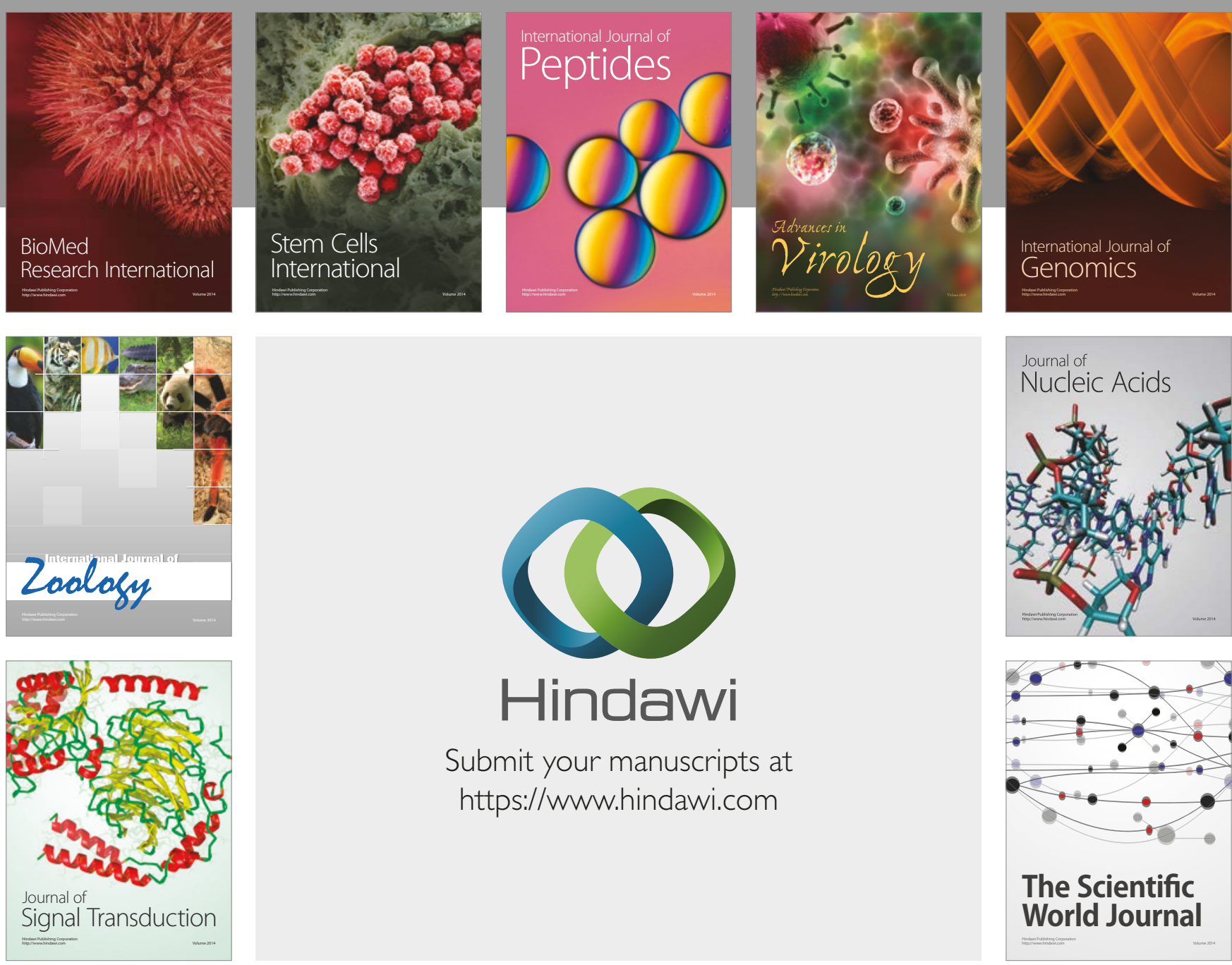

Submit your manuscripts at

https://www.hindawi.com
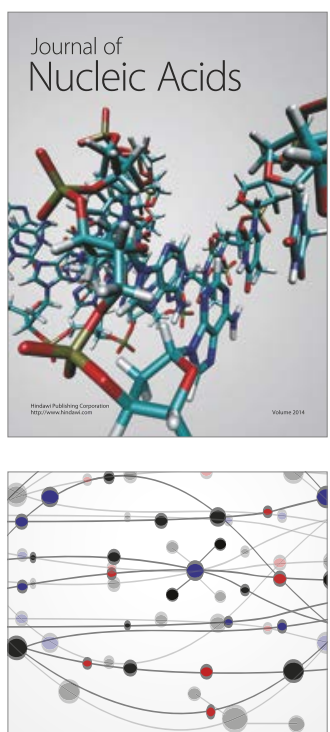

The Scientific World Journal

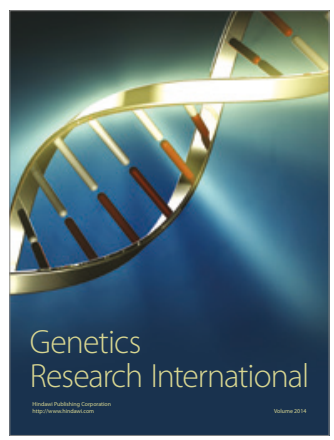

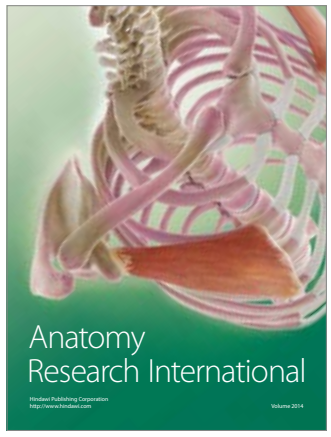

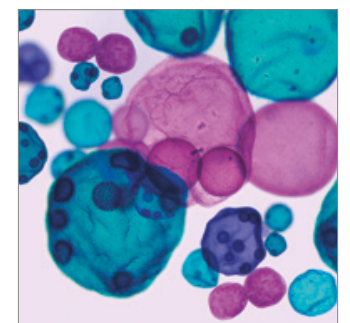

International Journal of Microbiology
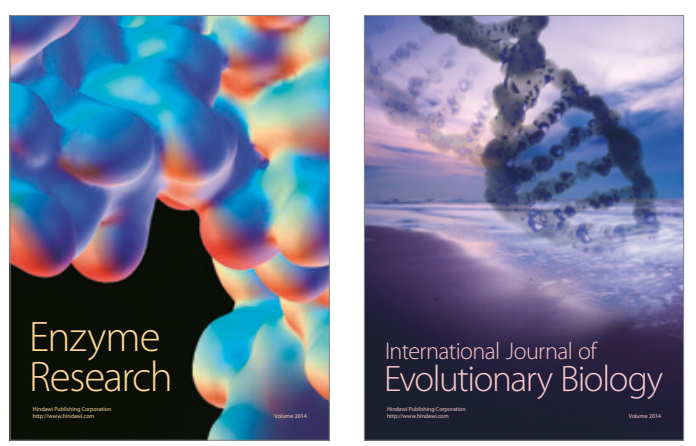
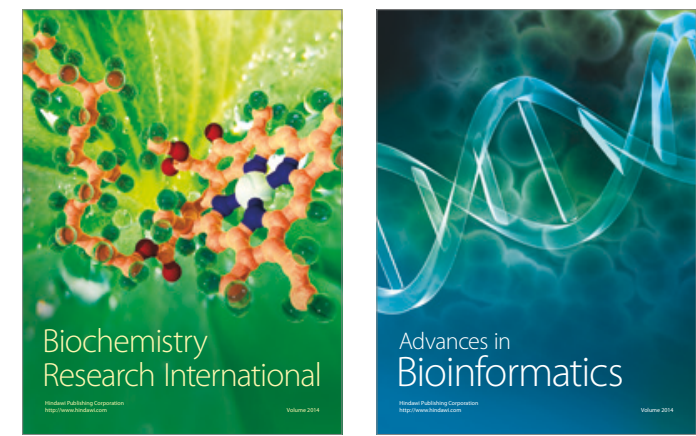

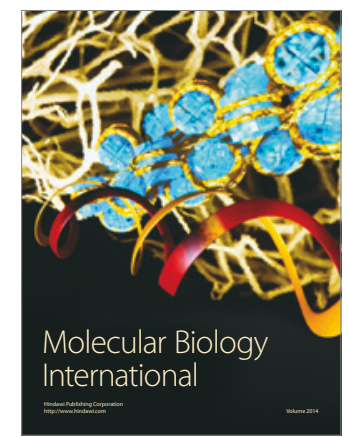

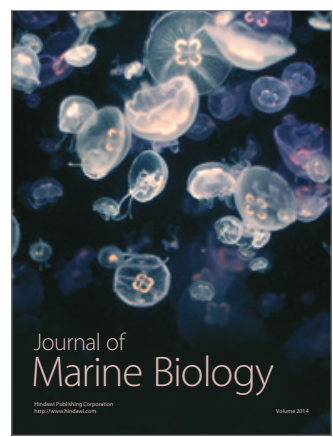

